\title{
The role of robotic technology in cardiac surgery
}

\author{
Elise Quint, Gayathri Sivakumar
}

\begin{abstract}
Minimally invasive robotic surgical techniques have been developed in order to improve patient outcomes and satisfaction. These minimally invasive techniques have been applied to numerous fields, including cardiac surgery. Currently, mitral valve repair and coronary artery bypass grafting are the most common procedures performed robotically. Numerous studies have shown that robotic technology provides similar outcomes to traditional surgery, which is much more invasive. However, there are numerous barriers to performing robotic surgery, including the cost of robotic systems and the steep learning curve associated with these systems. It is predicted that the indications for robotic cardiac surgery will increase as these limitations are addressed.
\end{abstract}

\section{INTRODUCTION}

The development and subsequent adoption of laparoscopic surgery in the 1980s and 1990s has encouraged the advancement of other minimally invasive surgical techniques. ${ }^{1}$ These techniques have been shown to improve patient outcomes and satisfaction and lead to shorter hospitalizations. ${ }^{1}$ This eventually led to the development of robotic surgery systems.

The most commonly used robotic system is the da Vinci surgical robot, which received its first FDA approval for use in surgery in 2000. ${ }^{1}$ However, the da Vinci system was not approved for use in cardiac procedures for several more years. ${ }^{1}$ The da Vinci system is completely controlled remotely by the surgeon. There are two main parts to the system: a bedside unit and a surgeon console. ${ }^{1}$ The bedside unit contains the endoscope with various cameras providing the surgeon with 3-dimensional vision, as well as several arms with miniaturized surgical instruments such as scalpel, scissors, forceps, and electrocautery. The surgeon console consists of video screens, hand controllers, and pedals for functions such as camera focus and electrocautery. ${ }^{1}$

There are numerous advantages to robotic surgery when compared with laparoscopy or open surgery. It provides 3-dimensional visualization of the surgical field, whereas laparoscopy only provides 2-dimensional vision. ${ }^{2}$ The hand movements used to operate the robotic system are more ergonomic and more closely approximate the range of motion of the human wrist. ${ }^{3}$ In laparoscopy, surgeons must move their hands in the opposite direction that they want the instrument to move, which can impair hand-eye coordination. ${ }^{2}$ Using the robot allows for more natural control of the instruments. In addition, the robot can scale down the surgeon's hand movements in order to perform very precise procedures. Finally, robots are able to minimize surgeon tremor. $^{3}$
This surgical innovation has been extended to many fields, including cardiac surgery. Most types of cardiac surgery have traditionally been performed through a large midline sternotomy incision with its attendant risk of complications such as bleeding and infection. ${ }^{1}$ There was naturally a strong push to apply any technology that could mitigate these risks, leading to the use of the first robotic cardiac surgeries in the late 1990s. ${ }^{1}$

\section{CURRENT ROLE OF ROBOTIC CARDIAC SURGERY}

The current use of robotic surgery varies greatly depending on procedure. Yanagawa et al estimate that the rate of robotic assisted cardiac surgeries increased from $0.057 \%$ to $0.390 \%$ from 2008 to 2011 in the US. ${ }^{4}$ While this is a large increase, it does not compare to the growth in other specialties, such as urology and gynecology. For example, the rate of robotic-assisted hysterectomy performed for benign disease increased from $0.5 \%$ to $9.5 \%$ from 2007 to $2010 .{ }^{5}$ Even more striking is the increase of robotic surgeries for radical prostatectomy, from $1 \%$ in 2001 to $40 \%$ in $2006 .{ }^{3}$ Described below are several cardiac surgeries where robotic use is highest. In Canada, availability of these procedures is generally limited to tertiary care hospitals.

\section{Valve surgery}

Robotic cardiac surgery was first applied to mitral valve repair, and this continues to be one of the largest uses of the technology. ${ }^{6,7}$ There are distinct advantages and disadvantages with this approach. Time on cardiopulmonary bypass may be longer for robotic surgeries; however, there several studies have shown that there is a significant reduction in complications which leads to a shorter length of hospital stay. ${ }^{8,9}$ The length of time the patient must spend on cardiopulmonary bypass is also decreasing as a result of technological advances. ${ }^{10}$ Relative contraindications to robotic mitral valve surgery include significant left ventricular dysfunction, pulmonary hypertension, and extensive annular calcification. ${ }^{11}$ The outcomes, including mortality, of minimally invasive mitral valve surgery appear to be comparable to that of mitral valve surgery with an open sternotomy. ${ }^{12}$

In addition to mitral valve repair, the tricuspid valve is sometimes repaired using the robot but often in conjunction with other procedures. For example, one group performed a series of concomitant tricuspid and mitral valve repair operations with an acceptable rate of complications. ${ }^{13}$

\section{Coronary artery bypass grafting (CABG)}

Coronary artery bypass grafting is used in place of percutaneous coronary intervention for certain patients who have atherosclerotic disease. There are various types of robot assisted CABG, which are referred to as Hybrid Coronary Revascularization (HCR) 
procedures. ${ }^{14}$ The goal of these minimally invasive approaches is to avoid sources of morbidity such as a full sternotomy, aortic clamping, and cardiopulmonary bypass. ${ }^{14}$ The least invasive type of HCR is referred to as totally endoscopic coronary artery bypass (TECAB). ${ }^{14}$ Other techniques may use the surgical robot but often involve a minithoracotomy, which is smaller than a sternotomy but larger than the endoscopic ports required for TECAB.

As in other types of robotic cardiac surgery, there are relative and absolute contraindications to TECAB, and therefore patients must be carefully selected for these surgeries. ${ }^{14}$ Absolute contraindications include patients with hemodynamic instability and severely impaired pulmonary function. Relative contraindications include the presence of pleural adhesions, significant space limitation (i.e. due to obesity or an enlarged heart), and chest deformities. In addition, up to $10 \%$ of patients need to be converted to an open sternotomy during surgery due to difficulties with the operation. ${ }^{14}$ Gasior et al compared HCR and standard CABG and found that the rate of death, MI, major bleeding, and repeat revascularization were equivalent in the two groups. ${ }^{15}$

\section{Other applications}

Robotic surgery has been successfully used to repair several other cardiac abnormalities, although these applications are less common than mitral valve repair and CABG. Atrial fibrillation ablation, patent ductus arteriosis closure, and atrial septal defect closure have all been performed via surgical robot. ${ }^{16-18}$

\section{DISCUSSION}

Current evidence shows the outcomes of robotic surgeries appear to be at least equivalent to outcomes of traditional surgeries. ${ }^{4}$ This raises questions as to why robotic cardiac surgery has not been more widely adopted, especially when compared to the adoption of this technology in other surgical specialties. Despite the many benefits of robotic surgery systems, the technology also has several limitations.

The cost of robotic surgery is significant and must be taken into account. First of all, acquiring a robot such as the da Vinci requires a large financial outlay which is simply out of reach at many Canadian hospitals. ${ }^{2}$ Secondly, the instruments can only be used a finite number of times before they must be replaced, adding additional costs to the surgery. ${ }^{1,19}$ These costs mean that in general, cardiac surgeries are more expensive when using a robot when compared to a laparoscopic approach. ${ }^{4}$

Another oft-cited explanation for the lack of adoption of robotic approaches is the steep learning curve associated with learning to perform these surgeries. ${ }^{14}$ Surgeons may only take on more complex procedures as their skill level increases. ${ }^{12}$ There are also technique limitations when using robotic surgery. While the da Vinci system is very ergonomic, one thing that has not been replicated in laparoscopic or robotic surgery is haptic (touch) feedback, although researchers are working to develop this technology. ${ }^{2}$ In addition, Canale et al cite the lack of an endoscopic surgical tradition in the cardiovascular surgery community which may lead to cultural barriers to the adaptation of this approach. ${ }^{19}$ Robotic surgical procedures also generally have longer operative times, which means that they use more operating room time when this resource is in short supply. ${ }^{14}$ However, some authors claim that with increased surgeon experience, operative time can approach that of cardiac surgeries involving open sternotomy. ${ }^{711}$

In addition to the above limitations, there are other surgical approaches that are even less invasive than robotic surgery. Procedures such as transcatheter aortic valve implantation, where an aortic valve is inserted through a catheter, are excellent options for certain patients and have become more common in recent years. ${ }^{20}$

In conclusion, the use of robotic techniques has been shown to be safe and effective in numerous types of cardiac surgeries. The main barrier to the use of robotic systems appears to be the high costs associated with performing these surgeries, and to a lesser extent, the extensive training required to master the robotic system. Certain questions remain to be answered with regards before robotic surgery becomes the standard of care for the above cardiac surgeries. While there is some robust existing data about, more randomized controlled trials could help clarify the role for robotic technology in cardiac surgery. While the adoption of this technique has thus far been relatively limited in cardiac surgery, it continues to increase. The broader applications of robotic technology will likely contribute to this approach having a larger impact on the field of cardiac surgery in the future.

\section{REFERENCES}

1. Woo YJ. Robotic cardiac surgery. Int J Med Robotics Comput Assist Surg. 2006 Sep;2:225-32. https://doi.org/10.1002/rcs.98

2. Lanfranco AR, Castellanos AE, Desai JP, et al. Robotic surgery: A current perspective. Ann. Surg. 2004;239(1):14-21. https://doi.org/10.1097/01. sla.0000103020.19595.7d

3. Leddy LS, Lendvay TS, Satava RM. Robotic surgery: Applications and cost effectiveness. Open Access Surg. 2010 Sep;3:99-107. https://doi.org/10.2147/OAS. $\underline{\mathrm{S} 10422}$

4. Yanagawa F, Perez M, Bell T, et al. Critical outcomes in nonrobotic vs roboticassisted cardiac surgery. JAMA Surgery. 2015 Aug;150(8):771-7. https://doi. org/10.1001/jamasurg.2015.1098

5. Wright JD, Ananth CV, Lewin SN, et al. Robotically assisted vs laparoscopic hysterectomy among women with benign gynecologic disease. JAMA. 2013 Feb;309(7):689-98. https://doi.org/10.1001/jama.2013.186

6. Carpenter A, Loulmet D, Aupècle B, et al. Computer assisted open heart surgery. First case operated on with success. C R Acad Sci III. 1998 May;321;437-42.

7. Bush B, Nifong LW, Alwair H, et al. Robotic mitral valve surgery-current status and future directions. Ann Cardiothorac Surg. 2013 Nov;2(6):814-17. https://doi. org/10.3978/j.issn.2225-319X.2013.10.04

8. Mihaljevic T, Jarrett CM, Gillinov AM, et al. Robotic repair of posterior mitral valve prolapse versus conventional approaches: potential realized. J Thorac Cardiovasc Surg. 2011 Jan;141(1):72-80. https://doi.org/10.1016/j.jtcvs.2010.09.008

9. Woo YJ, Nacke EA. Robotic minimally invasive mitral valve reconstruction yields less blood product transfusion and shorter length of stay. Surgery. 2006 Aug;140:263-7. https://doi.org/10.1016/j.jtcvs.2010.09.008

10. Nifong LW, Alwair H, Chitwood WR. Significant reduction in operative times using the Cor KnotTM device in robot-assisted mitral valve repair. Presented at: 12th Annual Meeting of the International Society for Minimally Invasive Cardiothoracic Surgery; 2013 Jun 12-15; Prague, Czech Republic. 
11. Gillinov AM, Suri R, Mick S, et al. Robotic mitral valve surgery: current limitations and future directions. Ann Cardiothorac Surg. 2016 Nov;5(6):573-6. https:/doi.org/10.21037/acs.2016.03.13

12. Cuartas MM, Javadikasgary H, Pfannmueller B, et al. Mitral valve repair: robotic and other minimally invasive approaches. Prog Cardiovasc Dis. 2017 Dec;60:394404. https://doi.org/10.1016/j.pcad.2017.11.002

13. Lewis CTP, Stephens RL, Tyndal CM, et al. Concomitant robotic mitral and tricuspid valve repair: Technique and early experience. Ann Thorac Surg. 2014 Mar;97(3):782-7. https://doi.org/10.1016/j. athoracsur.2013.09.049

14. Ejiofor JI, Leacche M, Byrne JG. Robotic CABG and hybrid approaches: the current landscape. Prog Cardiovasc Dis. 2015 Nov-Dec;58(3):356-64. https://doi. org/10.1016/i.pcad.2015.08.012

15. Gasior M, Zembala MO, Tajstra M, et al. Hybrid revascularization for multivessel coronary artery disease. J Am Coll Cardiol Intv. 2014 Nov;7(11):1277-83. https:// doi.org/10.1016/j.jcin.2014.05.025

16. Gerosa G, Bianco R, Buja G, et al. Totally endoscopic robotic-guided pulmonary veins ablation: an alternative method for the treatment of atrial fibrillation. Eur J Cardiothorac Surg. 2004 Aug;26(2):450-2. https://doi.org/10.1016/i. ejcts.2004.04.043

17. Le Bret E, Papadatos S, Folliguet T, et al. Interruption of patent ductus arteriosus in children: robotically assisted versus videothorascopic surgery. J Thorac Cardiovasc Surg. 2002 May;123(5):973-6. https://doi.org/10.1067/ mtc. 2002.121049

18. Ishikawa N, Watanabe G. Robot-assisted cardiac surgery. Ann Thorac Cardiovasc Surg. 2015;21(4):322-8. https://doi.org/10.5761/atcs.ra.15-00145

19. Canale LS, Mick S, Mihaljevic T, et al. Robotically assisted totally endoscopic coronary artery bypass surgery. J Thorac Dis. 2013 Nov;5 Suppl 6:S641-9. https:// doi.org/10.3978/j.issn.2072-1439.2013.10.19

20. Cribier A. Development of transcatheter aortic valve implantation (TAVI): a 20year odyssey. Arch Cardiovasc Dis. 2012;105(3):146-52. https://doi.org/10.1016/i. acvd.2012.01.005 